\title{
Computerizing the perception laboratory: Surviving the transition
}

\author{
STANLEY COREN \\ University of British Columbia, Vancouver, British Columbia, Canada
}

\begin{abstract}
A number of problems may be encountered during the transition to a computerized perception laboratory. Although some of these problems are of a technological nature, others have to do with the psychology of the investigator. These problems are discussed in the context of actual incidents taken from various research facilities, and some suggestions for avoiding common difficulties are presented.
\end{abstract}

Computers have caused a revolution in the operation of many, if not most, laboratories in which perception is studied. Much has been written about how investigators should go about modifying laboratories to make use of this new technology. It is easy to find enthusiastic descriptions of what you can expect your computer to do, how you can interface your computer with other equipment, and how you can utilize or develop specialized software. Articles, such as that written by Uttal (1972), that warn that the process of computerization of a research facility may be accompanied by certain avoidable pitfalls, are rare. This is unfortunate, as can be attested to by the many laboratories suffering from computer paralysis, a technologically induced disease that occurs in researchers who have not been inoculated against some of the inevitable problems that attend the transition to a computerized laboratory. This article presents some suggestions that have emerged from the experiences of several laboratories that went through the process of computerization and survived.

There are many reasons for the computerization of a laboratory. The most obvious is that a particular experiment or research paradigm requires the investigator to consider the introduction of this new technology. The reality is that most researchers begin to computerize their research because it seems to be about time. In our own case, the laboratory had been running quite efficiently and successfully for many years. Our equipment was not very elegant, consisting mostly of timers, projectors, a tachistoscope, and a collection of wood and cardboard contraptions that served as stimulus displays, such as adjustable illusion configurations. Visitors to our laboratory often expressed sympathy and assured me that everything I was currently doing could be done more efficiently, faster, and with lower labor expenditure via computer. My visits to other facilities reinforced the feeling that we were tech-

This research was supported in part by grants from the Natural Sciences and Engineering Research Council of Canada. I would like to thank Lawrence $\mathbf{M}$. Ward, who read an early version of this manuscript.

The author's address is: Psychology Department, University of British Columbia, Vancouver, British Columbia, Canada V6T 1W5. nologically lagging, especially when I was shown examples of computers in action (although, as I look back, the actual experiment was almost always in the process of being set up, to be run "after we work out a few bugs"). I suppose it was more from social pressure than from a sense of need that I finally gave in and purchased the first of a series of computers. Once it had arrived, I found that we had a lot to learn if we were to survive the transition. As is the case for most investigators beginning to computerize a laboratory, although we may have been sophisticated in terms of perceptual methodologies, we were computer naive. We fell into traps that are often so obvious to the expert that he/she does not feel it necessary to warn the neophyte. To save some future novices from similar pain, I would like to outline some of these problem areas.

\section{MICRO- VERSUS MINICOMPUTER}

The first question that has to be answered is what type of computer to purchase. When we sought advice we were often told that we should always get the most powerful computer (or the largest memory or the largest-capacity disk drives) we could afford. We were assured that this would reduce the time and cost of each calculation and provide functionally unlimited memory capacity, among other benefits. According to this credo, if you can afford it, you should get a mini (or, if you are really well off, even a mainframe). For the perceptual laboratory, however, this may well prove to be a myth.

Consider the researcher who acceded to such advice and bought the largest minicomputer he could afford. To allow several experiments to be performed simultaneously, he decided to time share over several research stations. His reasoning was that the main processor was so powerful that it could easily handle the slow input and output requirements of human subjects participating in a number of experiments. Problems immediately arose. To begin with, networking costs money. In this case, each additional station cost close to $40 \%$ of the price of the original computer. In addition, there were software over- 
head costs, since the networking itself required additional programming. The fatal flaw in the whole scheme, however, resided in the fact that, even with a fairly powerful unit, the nature of experimental control requires a number of real-time computations. In one instance, the researcher found that a particular experimental procedure would randomly lock out all stations other than the one on which it was being run. Although sophisticated computer users are probably well aware of this possibility, by definition, a researcher embarking on his/her first attempt at computerization is quite naive. This researcher solved his problem by purchasing a microcomputer for that one experiment alone, thus freeing the other stations for time sharing. It is interesting to note that this microcomputer costs less than half of what any one added station on the network costs.

Today many highly efficient microcomputers are available. Many have good graphics capabilities for display purposes, some have color capability, and random-access memory sizes routinely run from $16 \mathrm{~K}$ to over $500 \mathrm{~K}$. For all but the most complex experimental control situations, these features are quite adequate. Moreover, they are extremely cost effective.

In addition to avoiding the cost and real-time access problems associated with a time-sharing network, there are other advantages to using a series of microcomputer stations. In a network, if the central computer (or hard disk) fails, all stations fail. In a series of microcomputer stations, only the one suffering from hardware difficulties ceases to function. All other stations remain operative. Furthermore, if the programming is compatible, the experiment can simply be transferred to one of the other stations.

Another advantage is the portability of the equipment. To move a microcomputer research station, you need only carry it from one place to another. If necessary, you can then use the same central processor to interface with a number of different specialized stimulus display units. In networking, you must make physical contact with the relatively immobile central computer. This often involves a maze of cables connecting one experimental station to another. As the length of the interconnecting cables increases, so does the vulnerability of the system. This fact became all too salient to one researcher, whose network became inoperative for almost a week. A rat (presumably an escapee from an undergraduate lab) with a taste for electrical insulation had shorted the line. The culprit was discovered only because of the scent emanating from its body after it had been electrocuted while nibbling a nearby power line. Other investigators have mentioned overzealous cleaning or maintenance personnel as being equally dangerous to extended networks.

Microcomputers also offer portability of expertise. If you select a common make of microcomputer, you quickly find that many other people who own similar machines have developed programs and programming expertise for the unit you have chosen. This can be of particular value in developing software for your own laboratory applica- tions. Routines can be borrowed and adapted from preexisting programs quite easily. Also, programming assistance is readily at hand, often from unexpected sources. A demonstration of this ambient expertise occurred in our lab. In an early stage of our computerization, we had developed a program to randomize conditions in blocks for a particular experiment. Although the program worked, when the number of conditions and blocks became large, it would sometimes take several minutes for each new randomization to be set up. The program was running (giving occasional indications on the screen to assure us that something was happening) when a group of high-school students toured our lab. One stopped and asked about the activity on the computer. When it was explained to him, he said, "I used to have time problems in a card-shuffling program, but they're easy to solve." He sat down confidently, listed the program, deleted and entered a few lines of code, and since then the program has run about a factor of 10 faster. The trick he taught us, which has since been incorporated into a number of other applications, would not have been available to us except for the fact that we were using a very common microcomputer and knowledge of how to program it was equally common.

\section{PROGRAMMING AND PROGRAMIMERS}

Unfortunately, regardless of the choice of computer, experiments are ultimately controlled by programs. The decision that must be made is whether the experimenter will do the programming or whether a programmer should be hired. This decision is not easy, since there are a number of advantages and drawbacks to both courses of action. It is usually believed that the major advantage to hiring a programmer is that it saves the time of the researcher, who then does not need to become proficient in the programming language or spend the many hours required for actual program production. The amount of time saved, however, may prove to be less than expected.

For programmers to work effectively, their instructions must be quite clear and detailed. It is truly remarkable how many components of any experimental paradigm we do not explicitly state, but take for granted. These range from obvious (to the experimenter) steps, such as the need to randomize or counterbalance stimulus sequences, to much more subtle, experiment-specific factors. Subtle but important factors can be overlooked by a programmer. For example, a colleague and I once employed a programmer to create a program that would measure decrement of the Müller-Lyer illusion. (This is the gradual reduction of illusion magnitude that is observed after viewing a figure for a few minutes.) The resultant program was quite beautiful. The illusion figures were clear, the adjustment procedures smooth and precise. The only problem was that when we used the initial version of the program, we got virtually no illusion decrement. This was puzzling, because the effect is so reliable that we have routinely used it as a demonstration experiment in undergraduate laboratories with primitive adjustable figures 
made from cardboard. Furthermore, the source of the problem was rather elusive. When we pretested the main program, we obtained the expected illusion reduction over the 5-min test period; however, in the actual experiment, our naive subjects simply did not obtain a reduction at all. The answer came while we were demonstrating the program to a visitor. For the purpose of demonstration, we used the entire program sequence, including the instructions to the subject. This segment contained full graphics that illustrated the figure, showed how the adjustments were to be made, gave a few practice runs, and so forth (much like an interactive movie). To our chagrin, it also contained nearly $3 \mathrm{~min}$ of exposure to the actual illusion configuration. It was during this instructional period that most of the illusion decrement was taking place, rather than during the actual experimental phase. Any perceptual researcher would have known to use a neutral control figure for this phase of the experiment, precisely to avoid such decrement prior to the actual measurements. We had forgotten that the programmer, whose expertise was in programming, could not be expected to know this "obvious" point of procedure. When we had indicated that the subjects would need instructions and a bit of practice prior to the experiment, the programmer, quite naturally, provided direct practice on the actual stimulus figure. In this painful way we learned that every aspect of the experiment must be spelled out in detail.

Many programmers go out of their way to make programs as artistic and elegant as possible. Although this often results in programs that are more efficient and aesthetically pleasing, there are occasions when elegance may invalidate the experimental procedure. One blatant instance of this involved a programmer who was enamored of menus. Before each phase of the experiment, a menu appeared, the experimenter selected the conditions for the next block of trials, and momentary highlighting confirmed the choice. The options set conditions for a signal indicating the side on which a flashed target would appear. These conditions could vary from always valid to always invalid, with several selectable intermediate probabilities. The experimenter monitored the experiment on his own screen, which presented the image of everything that the subject saw. Unfortunately, the linking of the experimenter's control monitor with the subject's display monitor meant that the subject also saw everything that the experimenter saw. The elegant menu that highlighted upcoming conditions also informed the subject as to the validity of the cue in the next block of trials! A less elegant display, which simply instructed the experimenter to enter the next stimulus condition number, quickly cured this problem.

Other cases are more subtle. For instance, a programmer working on a routine for analyzing eye movement inputs was quite upset by the "shaggy" shape of the inputs, due to noise from resting nystagmus, microsaccades, and so forth. He set about cleaning up the results, in order to more clearly display the actual saccades. The problem was that his smoothing routine worked too well. His program utilized a displacement window that was large enough to completely obliterate all of the small corrective eyeflicks that the experimenter was interested in studying. Unfortunately, the programmer did not bother to inform the experimenter of this modification, which the programmer considered merely a cosmetic way to make the outputs more elegant.

Since programmers consider their input to be creative in nature, they often vary or change the program or the hardware requirements simply to try something new. In another eye movement study, the experimenter felt that the subject would benefit from a warning signal indicating that the stimulus sequence was about to begin. The programmer was intrigued by a newly acquired tone generator and decided (simply to vary the routine) to see if he could interface the stimulus control program with it. After spending several days (paid for at the usual programming rates) learning how to interface the tone generator, he set up an auditory "bleep" to signify the beginning of each trial. The problem was that the speaker was set up beside the screen, causing a large number of trials to be biased by the subject's involuntary eyeflicks in the direction of the sound source. Less aesthetic, more rapidly programmed signals, such as flashing the word "ready" or blinking the fixation point, would not have led to this contamination.

It might be argued that the above problems would not have occurred had the experiment been better supervised. Actually, such instances are common, because of a subtle psychological shift that frequently takes place during the process of computerization. The program is no longer the tool of the experimenter; it becomes the property of the programmer. In this role reversal, the programmer develops a feeling of control, in which the experimenter often acquiesces by ceding the right to make certain decisions. Perhaps the most common phrases the experimenter hears from the programmer at this stage are "You don't want to do it that way," and "There has to be a more elegant way to do that." By this time the program is usually partly written, and the experimenter is often estranged from its inner workings. The experimenter's resultant sense of insecurity and lack of control is then apt to lead to his/her further acquiescence and a final program that does what the programmer wants it to do, but not exactly what the experimenter wants it to do. It may work well enough that the experimenter feels that further change is not worth the effort; however, the fact that it is not exactly what the experimenter wanted clearly indicates that it was the programmer who had the final say, despite the fact that he/she may have had no training in sensory research.

One way to avoid such problems, of course, is for the experimenter to do the programming. At the very least, he/she should learn enough about programming to be able to globally understand what is occurring at each point in the program. Within this context, it is important to require the programmer to document each step of the program, 
with ample comments and remarks. Programmers do not seem to like to do this. They often argue that remarks take up memory, and hence slow the operation of the program. This is true; however, it is quite easy to keep two versions of each program. The first contains full documentation, and the second, stripped of remarks, is actually used for running the experiment. Any changes or modifications, of course, are made to both versions. Full documentation of each program protects the experimenter in a variety of ways. First, should the programmer leave, the program can be transferred to another, who stands a chance of continuing the work without spending weeks or months trying to trace the idiosyncrasies of the original programmer. Second, full documentation allows the experimenter to look through the program and find out what is being done for any particular function, without the necessity of fully decoding the main body. Third, it enables the experimenter to immediately find subroutines that are usable in other experiments with little modification. Even if the experimenter is the progammer, it is useful to document for these reasons.

\section{TOO MUCH OF A GOOD THING?}

Logic suggests that the process of computerization should be gradual, perhaps beginning with the use of the computer to relieve some of the drudgery of routine tasks. If the steps are repetitive and the number of opportunities for error are great (as in scoring the FarnsworthMunsell 100-Hue test for color discrimination), the computer appears to be the ideal solution (e.g., Coren, 1986). However, such gradual immersion into computerization seems to be the exception, rather than the rule. Many researchers, once they begin the conversion to computers, become overly enthusiastic and decide to computerize everything: All experiments must be run under computer control; all data will be analyzed on-line, stored on diskette for later analysis, or exported to the mainframe; all apparatus must be interfaced with the computer(s).

One example of total computerization comes from a laboratory that was conducting a series of color-naming experiments. The researchers chose to place all aspects of the experiments under computer control, so that no experimenter intervention was needed. The subjects were undergraduates enrolled in an introductory psychology course, who participated for course credit. Each subject picked up from a secretary a punched card that contained an experiment number, a subject number, and condition specifications. For each of four sessions, the subject simply came to the testing site at the arranged time, inserted the card indicating condition and experiment into the computer, and judged the stimuli according to instructions provided by the computer. At the end of each session the computer dispensed a voucher crediting the subject with an hour's participation; the subject then gave the card to a teaching assistant. The beauty of this system is that the experimenter never has to be physically present or actually see a subject. The problem with this system is that the experimenter is not physically present and never sees the subjects. This is a problem because of the nature of subjects and the nature of equipment. Let us consider each factor in turn.

One graduate student, whose thesis data was being collected via this system, was looking at the summary data output for his experiment. As he scanned the numbers, he noticed one subject whose data pattern indicated a color deficiency (probably deuteranomaly). This pattern was evident on Days 1, 2, and 4; however, it was not apparent in the Day 3 data. Surprised by this result, the graduate student contacted the subject to find out whether he may have been using tinted eyeglasses, or some other strategy to overcome his color weakness, on Day 3. The subject (who fortunately was honest) admitted that on Day 3, a friend of his had taken his place, and later that day he had filled in for his friend. "After, all, we were both on Day 3, so it shouldn't make any difference," he explained. Then, in a statement that cast doubt on the validity of the whole year's enterprise, he added, "A lot of us do this when the sessions are at inconvenient times."

Now aware that totally automatic operation might present some problems, the graduate student alerted the others in his lab. They began to spot-check their experiments to see if all was going well. One, who was now monitoring on a daily basis, noted that one subject's data seemed rather random relative to the others. To see whether she misunderstood the instructions or lacked English fluency, he came to the lab during her next session. Peeking into the experimental booth, he saw the subject comfortably filing her fingernails. At the auditory signal to judge the color, she would sometimes glance up at the screen and respond, and at other times would merely randomly touch one or two of the response buttons to advance the program.

The two incidents reported above suggest that subjects may view computer-run experiments differently than they view experiments run by a human researcher. The completely computerized experiment often conveys a feeling of impersonality, perhaps a sense of the unimportance of personal involvement in the task. On the other hand, the presence of a human experimenter, even one who serves as a passive monitor, can serve as a social motivator, a check of subject behaviors in the experimental setting, and a means of providing c'arification and information during data collection. Another point made by the above examples is the importance of monitoring individual subject and session data. If all of these data had been simply entered onto disk and then globally processed, it would have been nearly impossible to detect that anything was going wrong with individual cases. Hence these incidents would have passed unnoticed.

Equipment, as well as subjects, can cause subtle problems in the completely computerized lab. One investigator was using a microcomputer to generate line figures and to take complexity and familiarity judgments. The program had been carefully pretested and the various conditions had been piloted. Following good programming 
procedures, the experimenter had made copies of the master disk and used these copies to actually run the experiment. However, the resultant data seemed a bit strange. One common stimulus (a square) was often judged to be of extremely high complexity, with low familiarity. The experimenter ran through the program, but nothing seemed amiss. Some time later, one subject asked how "that weird figure" related to the others. Then he sketched a pattern that had not been programmed. The experimenter immediately rechecked the actual diskettes used with the subjects, and found that one of the three copies taken off the master had lost the bit of information that determined the starting point from which the square was drawn. This caused an asymmetric figure with a starting point off screen to be drawn whenever a square was called for. The other two copies were quite sound, but unfortunately, since no one had noted which copy had been booted for which subjects, the entire study had to be rerun.

Hardware has an annoying habit of failing in the most covert fashion, and in the absence of continuous monitoring, such failures are often quite costly. In one study, subjects were occasionally cued as to where a critical stimulus would appear by a brief flash of an LED. The LEDs were arranged in a ring around the monitor on which the target was displayed. The data suggested that cues to the left (opposite the normal reading direction) had virtually no effect on target localization. The data were actually in the process of being written up when a technician noticed that the LED for the leftmost cue was not lighting. Because the experimenter had yielded to the belief that there is no need to continuously monitor an experiment under complete computer control once it is up and running, it was impossible to determine when the LED had failed. A test of a few subjects after the apparatus was repaired suggested that the failure was quite early, since the leftmost cue turned out to be quite effective when the LED delivering it actually came on at the appropriate time. Daily monitoring, or a peripheral equipment-checking routine each time the program was booted, could have avoided this difficulty.

Another problem with total computerization is that some experiments are simply not suitable for computer control: the cost is too high and the benefits are nonexistent. An example of this type of experiment is the illusion decrement study mentioned earlier. Prior to the arrival of our computer, we had routinely run illusion decrement studies using adjustable illusion configurations made of cardboard. They work well, but are not very impressive looking. Of course, a separate apparatus is needed for each illusion array, or each illusion variant. We decided to computerize this entire set of experiments, reasoning that we could simply program the particular configuration that we desired, and run all variants directly on the screen. The program took close to 3 months to write. The graphics and data handling aspects were much more complex than we had thought they would be, but eventually (after our programmer was wealthy enough to take the holiday that I have often dreamed about) the program was finished. It was a beautiful program, it worked well, and it was quite impressive. Even today we use it-not for actual experimentation, but to impress visitors to the lab. The computer program did nothing the old cardboard apparatus didn't already do. Each version of the cardboard apparatus takes about 15 to 20 min to create, which is about the time it takes to reconfigure the program for a new illusion variant. The cardboard apparatus does not have automatic data entry capacity, but a pencil and a data sheet are adequate to enter one number every $60 \mathrm{sec}$ for a 5-min period. Our research assistants like the old apparatus better, claiming that subjects are less intimidated by it. Furthermore, it is totally portable, taking only seconds to move from one room to another, and no set-up time is required.

Analogous to the urge to computerize all aspects of experimental control is the urge to utilize the full capacity of the computerized system by collecting every scrap of data obtainable. The rationale for this is that the computer has this extra capacity, and the additional information may prove to be useful. One computerized reaction-time experiment was designed to determine whether there were latency differences between the dominant and nondominant eye, and whether there were interactions with handedness. Thus, to answer the theoretical questions, a maximum of 12 numbers were needed (i.e., the mean reaction time, variability, and error rate for each handeye combination). The experimenters, however, decided that since this experiment was under computer control, it might be useful to have all of the data. Thus, trial number, reaction time, stimulus presented, response selected, correct versus incorrect flag, markers indicating hand and eye used, interstimulus interval, interval between response and next stimulus presentation, and two other variables that have now slipped my mind but which pertained to the warning signal were recorded for each trial. Given that there were over 2,000 trials per subject, this represented a massive body of data. Extra memory had to be purchased for the microcomputer, and the data files of each subject required several diskettes. Needless to say, this quantity of material was far too large and complex for analysis on a small machine; hence, the material had to be exported to the mainframe and subjected to extensive processing, which took much programming time and effort. Ultimately, the researchers recognized that this number of variables was simply too large to deal with in any meaningful conceptual framework. The data were then "stripped" and mean reaction times, variability, and error scores were extracted, and a paper was presented based upon these data alone. Had these 12 numbers been targeted at the outset, even the limited microcomputer used in the study could have extracted them on-line. Because of the time, effort, and money that went into the initial data acquisition, the remaining data were kept somewhere on tape just in case they are needed, which we presume will not be during this lifetime. 


\section{THE LIVEWARE INTERFACE}

Perhaps the best solution to the tendency to overcomputerize lies in the recognition that the ultimate goal ought to be a computer-assisted, rather than a totally computerrun, laboratory. Unfortunately, this recognition often does not come until after the initial enthusiasm for computerizing everything has dissipated and the researcher takes a hard look at the amount of money and lost experimental time the transition has cost. Our own conversion to computer assistance, rather than total computerization, came about through chance.

While visiting one laboratory, we encountered a study of the visibility of computer-generated sinusoidal gratings. The study used a psychophysical technique called Parameter Estimation by Sequential Testing, or PEST (Cavanagh \& Anstis, 1980; Taylor \& Creelman, 1963), which takes advantage of the computer's memory and computational capacity. This method uses two intermixed staircase procedures (one ascending, one descending), and the step size for the threshold variable (in this experiment, contrast ratio) is halved each time the subject's response on a given staircase changes (in this experiment, from "visible" to "not visible," or vice versa). The procedure is quite efficient, and it seemed suitable for a study we were considering that pertained to intermittent stimulation thresholds. We decided to implement it in our lab. There were complications, however. In the setting in which we had seen this procedure used, the stimuli were generated on a CRT. For our purposes this was impractical, because, among other considerations, we wished to control the luminance over a very broad range, and we wished to have full color control. This ruled out a simple TV monitor or CRT setup. We already owned a rather elegant four-channel tachistoscope, which contained full electronic control of a large number of variables, and since our first study involved few stimulus changes but much varying of the flicker rates, it seemed reasonable to try to design an interface that would place the tachistoscope under direct computer control. When we inquired about cost and time, we found that the price would be well over a thousand dollars ("if there are no snags") and the conversion would take about a month ("maybe a bit more"). Both cost and time seemed a bit excessive.

I was once told that during the early stages of research on factor analysis (before the computer era), the difficulty of a problem was determined in terms of "clerk hours." These represented the number of hours of computation that clerks would spend on the problem. Multiplying the hourly pay rate for a clerk by the number of clerk hours required gave a cost estimate. When considering the computerization of a lab, it is reasonable to apply a similar principle. How many research-assistant hours will this procedure save? Simply multiply the research assistant's wage by the number of hours expected to be saved to determine a figure that can be used to estimate whether computerization is cost effective. In this instance, we were looking at an interfacing cost that would be the equivalent of nearly 200 research-assistant hours, which seemed far in excess of the amount of time that would actually be saved by constructing the interface.

Simple analysis of the structure of the problem provided us with a method of obtaining the benefits of the computer staircase tracking with minimal hardware cost. This method involves the use of a liveware interface (in which hardware is the machine, software is the program, and liveware is a human operator, technician, or research assistant). The major function of the computer is to keep track of the conditions and the subjects' responses and to determine the next stimulus in the sequence. The sole purpose of the interface is to convey this stimulus selection information to the display device and to trigger the next stimulus. We therefore substitute the liveware interface for the hardware interface. In other words, the PEST procedure is programmed into a microcomputer. The display screen indicates the starting stimulus setting, which is set by the research assistant. The subject's response is indicated to the computer, which then specifies the next value or stimulus. The computer thus keeps track of all computational material, responses, trial numbers, and so forth, and informs the researcher when the stimulus cards are to be changed and when the test session is completed. For the researcher the actual data acquisition is thus a bit boring (although I can think of no psychophysical testing procedure that does not share this characteristic during testing); however, the chance of error is minimal. Furthermore, complex analysis and real-time control are possible without the intervention of additional hardware. The presence of the experimenter also serves as a motivator for the subjects, and as a check on their understanding of the instructions (and the possibility of aberrant behaviors as well).

It is a short step from the concept of the liveware interface to that of the liveware peripheral. In our lab this came about as, in a number of studies, we began to utilize the computer to measure reaction times, cumulative response times, visibility times, and so forth (the computer is probably the ultimate evolutionary stage of the old-fashioned stopwatch). Each of these experimental setups involved a different testing station and was interfaced (usually at least partially via liveware) to some display apparatus. The problem arose at the end of each experiment, when it came to determining the final data for each subject. A number of options existed. The first involved writing the data to disk. This is useful if the data can then be directly utilized in some statistical analysis program. An alternative form of hard copy is a printed output. However, the cost of a printer for each experimental station seems unwarranted; hence, the liveware peripheral is used. At the end of each experimental session, at the researcher's command, a summary data screen is called up. Usually, the data consist of fewer than a dozen numbers (means, standard deviations, ranges, etc.-perhaps one per condition or stimulus). The researcher has a data sheet that is set up exactly like the screen in terms of labels and spatial 
layout; the researcher merely fills in the numbers displayed on the screen. The data sheets can be spirit duplicated at virtually no cost, and the time taken to fill in the numbers is on the order of a minute. It takes a lot of minutes, even at professorial salaries, to render an additional printer cost-effective under these circumstances.

There is another gain, equally important, from the use of a liveware peripheral. When a researcher acts as a liveware peripheral (in this case, as a printer), he/she must look at the data on a subject-by-subject basis. This means that if something strange appears in the data, the subject is usually still near enough to query. Usually the something strange translates into misunderstood instructions or a hardware failure at the stimulus delivery end. Either of these problems, if not caught early enough, can invalidate an experiment. When the data are easily and automatically stored on disk, the tendency is not to check after each session. When a human being must write down the data, the checking comes automatically, and with it comes a free insurance policy.

\section{A FINAL SURVIVAL THOUGHT}

I have tried to point out a number of potential problems and pitfalls that might be encountered during computerization of a perception laboratory. Most share two causes. The first is associated with expecting too much. This often leads the researcher to attempt to computerize aspects of the lab that will not benefit from the technology, to col- lect too much unneeded data, or to begin with too large a machine. The researcher must reorganize his/her thoughts so that the goal is to assist laboratory operations with the computer, not to cede laboratory functions to the machine. The second problem comes from a failure to recognize that the most flexible and easily programmed piece of equipment in the laboratory is the human researcher. Using him or her as an integral part of the computer-controlled operation of your lab will save money and time, and will ensure higher quality data. Give the computer the task of tending to the repetitive computation and monitoring, but do not expect it to take over the full functioning of the laboratory. Start small, and see if a larger role for the computer is desirable. This will maximize the likelihood that your own laboratory will avoid the perils of computer paralysis in its many manifestations.

\section{REFERENCES}

Coren, S. (1986). The Farnsworth-Munsell 100-hue test: A BASIC program for scoring and analysis. Behavior Research Methods, Instruments, \& Computers, 18, 323-324.

Cavanagh, P., Anstis, S. M. (1980). Visual psychophysics on the APPLE II: Getting started. Behavior Research Methods \& Instrumentation, 12, 614-626.

Taylor, M. M., Creelman, C. D. (1963). PEST: Efficient estimates on probability functions. Journal of the Acoustical Society of America, 41, 782-787.

Utral, W. R. (1972). Misuse, abuse, overuse, and unuse of on-line computer facilities by psychologists. Behavior Research Methods \& Instrumentation, 4, 59-61. 